TENDENCIAS

Revista de la Facultad de Ciencias

Económicas y Administrativas.

Universidad de Nariño

ISSN-E 2539-0554

Vol. XXI No. 1 - 1er Semestre 2020,

Enero - Junio - Páginas 110-129

\title{
LA LÚDICA Y EL DISEÑO, UN ESCENARIO ECONÓMICO EMERGENTE EN EL CONTEXTO NACIONAL Y REGIONAL
}

\author{
PLAYFULNESS AND DESIGN, AN EMERGING ECONOMIC SCENARIO IN THE \\ NATIONAL AND REGIONAL CONTEXT
}

\section{LÚDICA E DESIGN, UM CENÁRIO ECONÔMICO EMERGENTE NO CONTEXTO NACIONAL E REGIONAL}

FRANCO CAÑÓN_Omar

Magíster en Diseño de la Universidad de Palermo. Docente tiempo completo, Universidad de Nariño. E-mail: francodenat@ hotmail.com,Colombia.

\section{Recibido: 4 de octubre de 2019}

Aprobado: 9 de junio de 2020

DOI: https://doi.org/10.22267/rtend.202101.129

\section{RESUMEN}

Históricamente las dinámicas propias de la lúdica fueron asociadas con los escenarios exclusivamente pedagógicos y didácticos. Actualmente la lúdica y sus mecánicas han logrado un espacio de consideraciones desde el diseño, el cual le ha permitido convertirse en un factor diferencial y alternativa en el desarrollo de productos, bienes y servicios en el escenario nacional y regional. El propósito de este artículo es evidenciar esos emergentes espacios de la lúdica como recurso del diseño en el ámbito económico, describiendo su importancia y la posibilidad que ofrece en aspectos como la innovación y la experiencia de usuario. Desde esta perspectiva, la concepción de un diseño con enfoque lúdico como plataforma metodológica se convierte en un campo amplio trazado por elementos transdisciplinares que sugieren la presencia activa de distintas perspectivas de investigación y métodos de acción. A partir de diferentes casos que resultan paradójicos e informes oficiales de entidades nacionales y regionales; se evidencia la emergencia de economías que consolidan sus 
actividades a partir de mecánicas lúdicas generadas desde el diseño. En suma, el presente artículo señala la incidencia actual de estas nuevas dinámicas comerciales apoyadas en las dinámicas de la lúdica y que tienen como propósito convertirse en alternativas en la diversificación de mercados y que inciden positivamente en el desarrollo del país y la región.

Palabras clave: lúdica, diseño, experiencia, economías emergentes, gamificación.

JEL: A12, A13, B21, L82, L83

\section{ABSTRACT}

Historically, the dynamics of play were associated with exclusively pedagogical and didactic settings. Currently the playful and its mechanics have achieved a space of considerations from the design, which has allowed it to become a differential and alternative factor in the development of products, goods and services on the national and regional stage. The purpose of this article is to highlight these emerging spaces of play as a resource of design in the economic field, describing their importance and the possibility it offers in aspects such as innovation and user experience. From this perspective, the conception of a design with a playful approach as a methodological platform becomes a broad field traced by transdisciplinary elements that suggest the active presence of different research perspectives and action methods. From different cases that are paradoxical and official reports from national and regional entities; the emergence of economies that consolidate their activities is evident from the playful mechanics generated from the design. In sum, this article points out the current incidence of these new commercial dynamics supported by the dynamics of leisure and which are intended to become alternatives in the diversification of markets and that positively affect the development of the country and the region.

Keywords: playful, design, experience, emerging economies, gamification.

JEL: A12, A13, B21, L82, L83

\section{RESUMO}

Historicamente, a dinâmica do brincar estava associada a cenários exclusivamente pedagógicos e didáticos. Atualmente, o lúdico e sua mecânica alcançaram um espaço de considerações a partir do 
design, o que lhe permitiu se tornar um fator diferencial e alternativo no desenvolvimento de produtos, bens e serviços no cenário nacional e regional. O objetivo deste artigo é destacar esses espaços emergentes de jogo como recurso de design no campo econômico, descrevendo sua importância e a possibilidade que ele oferece em aspectos como inovação e experiência do usuário. Nessa perspectiva, a concepção de um design com abordagem lúdica como plataforma metodológica torna-se um amplo campo traçado por elementos transdisciplinares que sugerem a presença ativa de diferentes perspectivas de pesquisa e métodos de ação. De diferentes casos que são paradoxais e relatórios oficiais de entidades nacionais e regionais; o surgimento de economias que consolidam suas atividades é evidente a partir da mecânica lúdica gerada a partir do design. Em suma, este artigo aponta a atual incidência dessas novas dinâmicas comerciais apoiadas pela dinâmica do lazer e que se destinam a se tornar alternativas na diversificação de mercados e que impactam positivamente o desenvolvimento do país e da região.

Palavras-chave: brincalhão, design, experiência, economias emergentes, gamification.

JEL: A12, A13, B21, L82, L83

\section{INTRODUCCIÓN}

En la cultura, el diseño actúa como eje articulador y optimizador de procesos comunicacionales que se dan en ella. De esta manera, el diseño acude a factores de función y forma, a la estética y discursos persuasivos que tienen objetivos específicos y buscan la aproximación de un público determinado a una intención de un emisor. Bajo esta premisa, la lúdica ha venido en las últimas décadas tomando especial relevancia como espacio convergente, interdisciplinario y complementario a diferentes disciplinas que encuentran en ésta y sus dinámicas; posibilidades de mediación en los discursos no solo pedagógicos, sino también en escenarios como el de las economías emergentes.

Desde esta perspectiva el diseño ha encontrado en los procesos de gamificación, derivados de la lúdica, un nuevo mediador entre esos pedidos sociales y los discursos de estas nuevas formas de consumo; ya no solo como un proceso lineal de persuasión en la compra o adquisición de un producto, bien o servicio, sino como un espacio de intervención experiencial asistido por el disfrute, la emocionalidad y la recreación. En el mundo, en Colombia y en especial en la ciudad de Pasto, estas 
dinámicas de gamificación, aunque no son referenciadas de manera formal, cada vez son más frecuentes y se articulan desde el diseño en las economías emergentes aportando al crecimiento de la región y el país.

\section{REFERENTES TEÓRICOS}

Una de las más relevantes características de la sociedad contemporánea es su creciente demanda de bienes y servicios. La sociedad del consumo, como fenómeno social puede establecer su génesis en el siglo XX; cuando las lógicas mercantiles, como consecuencia directa de las dinámicas del capitalismo y los desarrollos de la mercadotecnia y la publicidad, encontraron escenarios para fomentar el consumo advirtiendo pedidos sociales que reclamaban atención a las nuevas necesidades sociales. Así los imperantes escenarios de consumo privilegiaron las acciones de la publicidad, el mercadeo y el diseño, construyendo una nueva forma de entendimiento y novedosas rutinas de tránsito y relación entre consumidor, mercado y la producción.

Durante la primera parte del siglo veinte, las sociedades se encontraban inmersas en un sistema social y económico que priorizaba la organización social del trabajo racional, el dinero y las ganancias obtenidas de las actividades de producción. En esencia, las maneras de producción de la sociedad industrial estaban constituidas a partir de la propiedad privada de los medios de producción. Desde esta perspectiva, la fuerza de trabajo era la única propiedad que poseía el trabajador, donde su actividad generaba un excedente que no se le reintegraba en su salario, sino por el contario era apropiada por la propiedad privada constituyéndose en capital (González, 2015).

Las dinámicas sociales actuales y los nuevos órdenes globales surgidos con el final de la segunda guerra mundial instalaron un escenario mundial donde la idea de bienestar social se convertía en los principios sociales de todo estado. De esta manera las construcciones de sistemas de consumo fueron entendidas desde el diseño como escenarios dinámicos de retroalimentación, dónde el consumidor asiste a esta lógica comercial, pero en todo caso, ya no de manera lineal y unidireccional sino comprendiendo que a cambio recibe otra serie de reconocimientos a otro tipo de necesidades como el status, el afecto, la experiencialidad y la autorrealización. 
Alvin Toffler (1980) expone en su libro, La tercera ola, justamente estas consideraciones en torno a cómo precisamente en espacios globalizados, el sistema comercial tendría que reinventarse, virar y encontrar nuevos discursos que involucraran a los ciudadanos, la emocionalidad en el consumo y el disfrute de la compra. Es así como la lúdica emerge como una actividad dotada de una resignificación social y ha encontrado en los distintos circuitos mercantiles y culturales un hábitat de crecimiento como concepto. En consecuencia, las empresas e instituciones de hoy, conscientes de la capacidad de respuesta positiva de los usuarios y clientes ante el estímulo propio de la mecánica lúdica, contemplan en sus estructuras de promoción o difusión, al juego como estructura experiencial y factor diferencial. Como resultado de lo anterior, desde las décadas finales del siglo XX se viene evolucionando en la concepción del producto actual, o al menos como se concibe desde su enunciación o en su planteamiento desde el diseño. Es decir, que ya la limitante de lo objetual, aunque sigue siendo primordial, no es necesariamente determinante en un proceso diseñístico; y sí lo es, el contexto de uso, su usabilidad y la experiencialidad; entendidos estos conceptos como elementos a incorporar en las políticas productivas modernas, como una nueva interpretación del consumidor y de sus anhelos, Krippenddorf (2016).

\section{METODOLOGÍA}

El presente artículo se enfocó, bajo el paradigma de investigación cualitativa, a partir de un proceso de análisis deductivo. Así mediante un proceso de identificación de tendencias del mercado, se

evidenciaron constantes prácticas que se inscriben dentro del concepto de economía naranja. Éstas dinámicas demostraron en los análisis y discusiones del presente texto, la vinculación de la lúdica como factor diferencial en la mayoría de los casos presentados. A partir de estos reconocimientos se realiza una discusión tendiente a la descripción y verificación de la lúdica y el diseño como escenarios activos de vinculación con las economías emergentes en el contexto regional y nacional, donde en una instancia posterior se relaciona el concepto de gamificación y sus mecánicas de acción previstas desde la disciplina del diseño. Seguidamente, se evidenciaron y consideraron casos de gamificación a nivel internacional que aportaron en la constricción del estado del arte. Dentro de la investigación se observaron las estadísticas ofrecidas por el Departamento Nacional de Estadística; DANE; específicamente el aporte al PIB Nacional de la economía naranja con el reporte del periodo comprendido entre 2014 y 2018. En dicho documento se encuentran referidos diferentes modelos 
económicos promovidos por la economía naranja, donde la lúdica preservó un lugar determínate como mecánica de acción y puesta en escena de estas economías. Con esta recolección de referentes e indicadores cuantitativos se procedió a reconocer e identificar casos nacionales que se crearon bajo las lógicas de gamificación.

Finalmente se analiza el despliegue de acciones regionales de empresas e iniciativas gubernamentales y académicas que evidencian la preocupación de este tipo de economías y la vinculación de la lúdica y las mecánicas del juego como activos importantes en este sector.

\section{DISCUSION}

\section{La Gamificación como mediadora en los nuevos consumos}

Una aproximación conceptual que permite la comprensión y relación entra la lúdica y el desarrollo económico la ofrece la Gamificación; término proveniente del anglosajón: Gamification, que define las ventajas de articular las mecánicas propias del juego en las estructuras empresariales e instituciones, diversificando su producción y servicios, Deterding (2011). Esta vinculación de dinámicas lúdicas como mecanismos de mejora empresarial y diversificación e innovación productiva en el segmento de las economías naranja se estructura con la asistencia del diseño.

El diseño como disciplina sistémica, ya sea visual, diseño de contenidos, diseño de productos o diseño industrial, en su acepción prefigurativa, contempla en los prototipos de productos, y servicios, dimensiones motivacionales de un consumidor que inciden en la experiencia de compra.

Justamente, es la lúdica un aspecto que se considera importante desde el diseño. El conocimiento sobre cómo la gente experimenta el uso de determinado objeto, o cómo percibe el contacto con una institución, hace parte de las características estudiadas en un proceso de diseño sistémico de productos, bienes y servicios. El diseño entendido como actividad prefigurativa atiende por un parte, las cuestiones específicamente relacionadas a función y forma, pero de otra parte tiene una indagación constante que se desvela en los procesos de ideación y creatividad, donde se busca precisar las condiciones que rodean el contexto de uso de un servicio o producto, Norman, (2005). 
Actualmente Muchas de estas consideraciones de diseño anexas en un producto o servicio, se suscitan en el placer de su uso, la sensorialidad, la emocionalidad, la armonía comunicacional, la navegación y estética virtual entre otras; condiciones estas últimas que permean muchas de las actividades de la economía naranja a propósito de economías emergentes.

Así, la lúdica desde el diseño, no debe entenderse exclusivamente desde el concepto de juego, pues es el diseño quien, mediante la incorporación y adaptación de lógicas y mecánicas propias de la lúdica, propicias formas de innovación, crecimiento y desarrollo empresarial.

Desde esta perspectiva el diseño aparece como una disciplina sistémica, de procesos estratégicos que brinda alternativas y soluciones en servicios y productos, vinculando las mecánicas propias de la lúdica, a partir de las estructuras de la Gamificación en las economías emergentes reconociendo particularidades en cada uno de los actores.

Desde el diseño diferentes metodologías han acompañado estas concepciones de producto intangible y de servicio personalizado. En efecto metodologías como la Co-creación tienen un enfoque participativo, donde diferentes actores como los mismos consumidores o usuarios intervienen de manera colaborativa en una etapa previa del diseño, definiendo las interacciones que se suscitan entre usuarios y el contexto que los rodea. Este enfoque metodológico permite advertir de fuentes primarias las características propias del contexto, de la usabilidad de un artefacto; concebida esta característica como la forma en que un consumidor manipula o usa un producto.

Otra de las metodologías que han estado acompañando estos diseños de contenidos e interacciones derivados de la lúdica en las economías emergentes es el Design Thinking. Este método de diseño, permite a al diseñador o grupo de diseñadores trabajar en equipo de manera abierta y propositiva. Así esta metodología, se convierte en una herramienta a través de la cual los usuarios son participes consolidando productos pensados desde los usuarios y para los usuarios, Serrano (2013).

Estas mediaciones del diseño entre economías emergentes y la lúdica a través de procesos de gamificación, optimizan los procesos, productos y servicios de las economías y empresas del sector (ver Figura 1). 


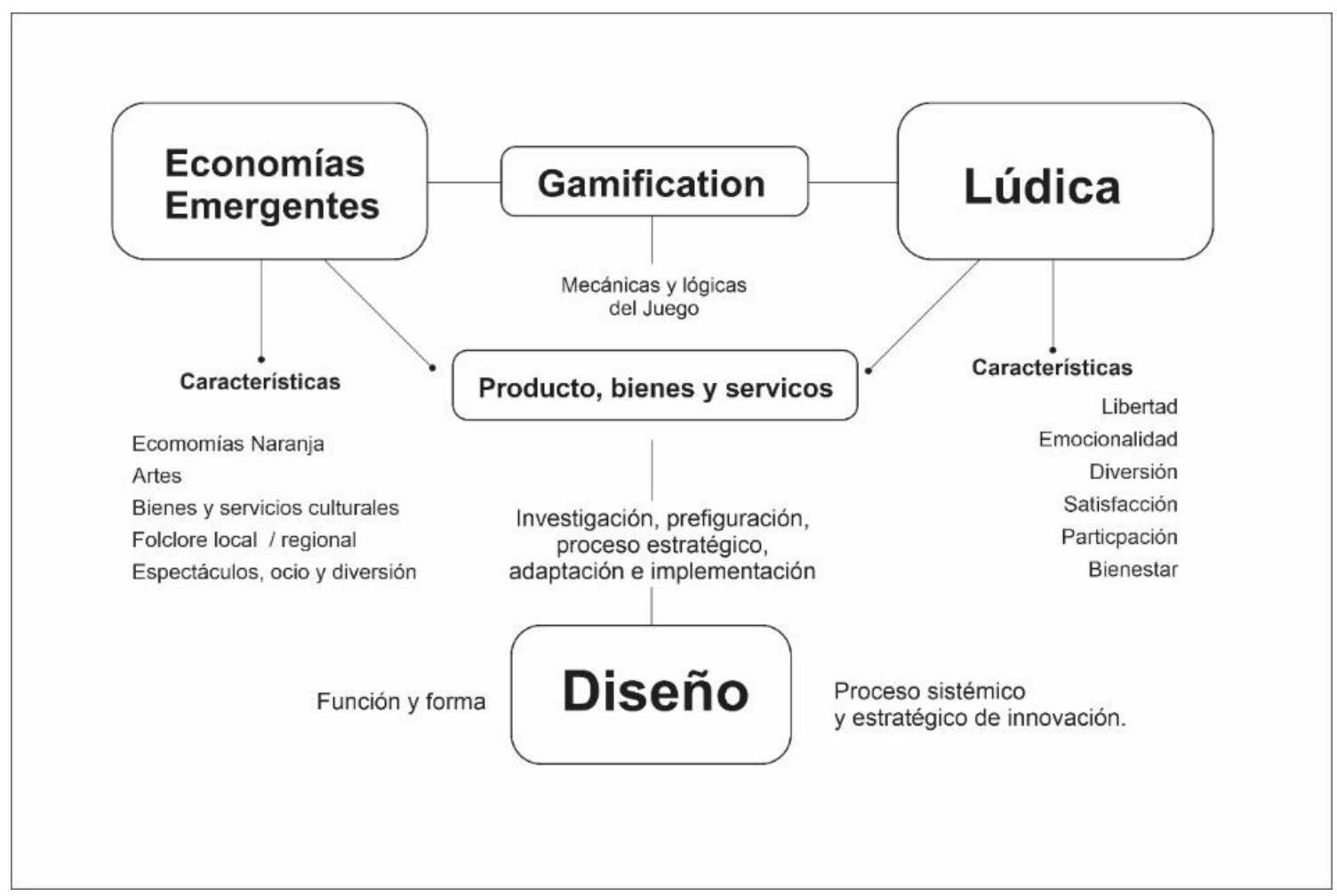

Figura 1. Estructura relacional entre la lúdica y economías emergentes.

Fuente: elaboración propia.

\section{La gamificación en las multinacionales}

Durante la última década, se han presentado casos paradigmáticos en los escenarios comerciales, que se pueden considerar pioneros en la vinculación de la lúdica como condicionante en sus relaciones con el consumidor. En 2009, The Fun Theory fue una iniciativa de la multinacional automotora Volkswagen, que a través de un domino web, buscaba la participación de los usuarios con la ideación de soluciones a problemáticas del orden internacional. La multinacional apostó por la diversión como una forma más humana de conectarse con sus usuarios, dándoles la posibilidad de expresar lo que pensaban de manera participativa y propositiva en la búsqueda de soluciones a distintas problemáticas. Más allá de la calidad de las soluciones la multinacional buscaba estrechar vínculos con sus usuarios, haciéndoles saber que lo que pensaban era importante, en este caso se evidenciaba la participación como un elemento propio de las mecánicas de juego provisto en un mecanismo de promoción empresarial. 
Estas mismas estrategias también se implementaron por parte de la casa deportiva NIKE. Específicamente en la aplicación $N I K E+$, en ella los usuarios compiten contra sí mismos y contra otros usuarios, comparando recorridos y rendimientos deportivos. De esta manera se activa la competencia, otra mecánica traída del juego e incorporada en una estrategia de imagen empresarial y de marca. Otras implementaciones de gamificación en contenidos, productos y servicios son las contempladas por entidades bancarias como BBVA. La multinacional implementó la estrategia de gamificación: BBVA game; que le permite al usuario aceptar retos virtuales y en recompensa obtiene puntos o premios. Mediante estas acciones la empresa familiariza a sus usuarios con los canales virtuales, liberando las sucursales físicas. En este caso, la noción de recompensa, aparece como una dinámica propia de la acción lúdica.

Quizá el actual emprendimiento más importante en este rubro es el Messi Park, espacio temático próximo a abrirse en Asia, que espera convertirse en un escenario de experiencialidad para los seguidores del futbolista y apasionados por ese deporte. Construido a partir de virtualizaciones y avatares de simulación, el Messi Park se articula dentro de los procesos de gamificación empresarial, donde el recorrido de sus instalaciones sugiere momentos de aproximación sensorial y desfrute estético.

Las anteriores estrategias implementadas por diferentes empresas evidencian como las mecánicas del juego como: la participación, la noción de recompensa o premio, la experiencialidad, fantasía y competencia, son características actuales de la forma en que un usuario experimenta una marca, un producto o servicio.

Es el juego un condicionante natural en el ser humano y su uso en las estrategias comerciales hoy es una constante, y es el diseño el encargado de asistir estos procesos de gamificación no solo en las multinacionales sino en economías emergentes del orden nacional y regional.

\section{El juego como acción ontológica}

El juego como condición connatural del ser humano se entiende como un principio necesario en el desarrollo individual, la conciencia colectiva, el enriquecimiento cultural y el funcionamiento de la 
sociedad. Sin el juego, las posibilidades de adaptación, renovación y desarrollo del individuo y grupal se hacen limitadas; ya que la disposición lúdica permite el pensamiento creativo, la imaginación, la construcción de relaciones y la acción transformativa, Piaget (19619. El juego, desde las perspectivas de entretenimiento físico y mental, la divergencia de pensamiento y la construcción de lo colectivo; es el medio propicio donde la humanidad se encuentra consigo misma para reconocer su libertad, sus capacidades presentes y las posibilidades futuras. Huizinga (2012) advierte sobre la condición del juego en nuestras acciones diarias y entendimiento de mundo, considerándolo como una acción inherente y connatural del ser humano y señalando las ventajas de abordar el juego como una instancia contenedora de posibilidades en la generación de cultura.

Entonces, se convierten en necesarias e inaplazables las preguntas acerca del involucramiento con los juegos, qué cantidad de tiempo y esfuerzo se debe dedicar a estos, cómo exponerse a los factores positivos del juego y cómo estas consideraciones hoy son provistas en las dinámicas mercantiles de las economías naranja. Los juegos en su condición multidimensional presentan una complejidad inherente para su abordaje académico debido a la cantidad de campos de conocimiento que intervienen en éste como la psicología cognitiva, la experiencialidad en la compra, el diseño ambiental y la narrativa comercial, solo para nombrar algunas. Estas consideraciones primarias ofrecen un panorama nutritivo en variables que hoy contempla la lúdica como mediadora del consumo.

Dentro de las distintas actividades que pueden incorporar una empresa para el incremento de sus ventas, la mejora de sus productos y la satisfacción de sus clientes, se encuentran las estrategias de mercadotecnia. A través de la difusión y promoción con tonos persuasivos e informativos es la publicidad la encargada de posicionar una empresa, que a su vez encuentra en el diseño; discursos estético-formales para optimizar dichos procesos. Pero también, a la luz de las nuevas tendencias del mercado, es necesario que el diseño atienda fenómenos del consumo, como el contexto de uso, la experiencia del usuario y los conceptos derivados de la usabilidad y funcionalidad.

Así el diseño se enfrenta con dos funciones sustanciales: por un lado, un enfoque histórico que atiende la función y la forma, y de otro lado el diseño como actor comunicacional determinante en procesos de persuasión, difusión y promoción advertidos en los planteamientos de la publicidad. 
Desde este planteamiento el diseño, en su acepción de actor comunicacional y optimizador de procesos de difusión, encuentra en las economías naranja un espacio de aporte concibiendo el tratamiento de las dinámicas de la lúdica y las mecánicas del juego como un activo en el diseño de estrategias de posicionamiento, mejora de contenidos, productos y servicios.

En cada una de los escenarios donde opera el mercadeo aparece de manera muy activa y visible el diseño como una actividad integradora que interviene desde terrenos como la construcción simbólica, en la noción de cultura y en los valores asociados a la identidad y su desarrollo. Estos movimientos en distintos escenarios le entregan al diseño grandes responsabilidades no solo formales sino culturales, Ramírez, (2011). En todo caso, el diseño como disciplina emergente encuentra su hábitat, sus espacios sociales de acción, justamente en terrenos como el mercadeo y la publicidad y los sistemas innovadores de promoción y difusión, donde la lúdica y las mecánicas derivadas del juego permiten otros tipos de aproximación con el usuario y consumidor.

La aparición de conceptos y campos como las experiencias lúdicas, la inmersión sensorial, el aprendizaje profundo o el deleite estético son características emergentes que condicionan la actividad productiva actual, así como la acción y el espacio social del diseño. Estas nuevas características se convierten también en nuevos nodos de vinculación e interacción con la sociedad. Así, estas nuevas rutas de entendimiento del diseño fijan encuentros con la usabilidad, el contexto de uso y valores motivacionales por parte del usuario en sintonía con realidades comerciales y culturales donde el usuario y la cultura demandan progresos constantes y nuevas plataformas caracterizadas por la experiencialidad, la sensorialidad, el gusto y la emocionalidad.

En consecuencia, la noción de diseño y específicamente la vinculación de la lúdica; encuentra en la cultura su punto de partida y llegada; es a partir del entendimiento de las manifestaciones culturales y sus lógicas derivadas, que el diseño presenta su acción profunda, evidenciada en la asistencia que presta a la dinámica de consumo actual donde se advierten procesos de autorrealización del individuo, la noción de identidad y las conexiones entre ciudadano, ciudad y sociedad, así como los circuitos y planteamientos de entornos comunicativos. Es justamente desde esta perspectiva que se manifiesta el 
entendimiento de la lúdica y sus mecánicas intervenidas desde el diseño como un factor y propulsor económico, social y gestor cultural.

En este proceso la economía naranja ha visibilizado las nuevas búsquedas y necesidades del ser humano y el diseño ha encontrado en la gamificación la posibilidad de conectar estas demandas sociales y culturales.

\section{La urgencia de modelos nacionales}

La implementación de metodologías de diseño integrales que convoquen la presencia colaborativa de diversas disciplinas, más que fijar procedimientos en cadena, implican reflexiones sobre el contexto actual del usuario, comprendiendo en profundidad las acciones humanas desde las comunidades, sus anhelos, sus pedidos sociales. Así una verdadera teoría del consumo se basará no en una teoría de las necesidades y de su satisfacción, sino en una teoría de la prestación social y de la significación (Baudrillard, 1974).

Desde la perspectiva de Dusell (1984) retoma relevancia la idea de definir claramente la estructura de prioridades proyectuales en los modelos de diseño regionales donde la competitividad e innovación surjan como resultado de incluir otras disciplinas en los procesos de diseño. Así también se infieren desde este enfoque que un país en vía de desarrollo, como es el caso de Colombia, los procesos de diseño deben tener en cuenta las necesidades culturales propias y sopesar estas particularidades derivadas en los procesos de producción. Lo advierte Simon (2006) cuando señala las disyuntivas constantes que enfrenta el diseño hoy y su tránsito entre las leyes que gobiernan los medios que constituyen la acción del diseño o las que gobiernan el entorno donde actúa. Desde esta concepción, es importante que el diseño enfrente ya no solo los retos que se desprenden de las dinámicas mercantiles (racionalidad limitada) sino que éste debe enfrentar resueltamente las características propias de los objetos, las acciones experienciales, el disfrute estético, las cualidades motivacionales de los usuarios y las características de sus contextos de uso.

Desde las perspectivas expuestas por estos autores se puede inferir que en el caso colombiano las disertaciones al respecto no se han dado con la profundidad esperada. Por el contrario, los estudios en nuestro contexto nacional y regional han estado pasivos en cuanto a reflexión sobre el diseño y lúdica, Rodríguez, (2007). Así las experiencias lúdicas no se han posicionado ni asociado de manera eficiente 
como mecanismo de aporte diferencial en el diseño con fines de difusión cultural y promoción social. No obstante, en el contexto nacional y regional se pueden citar algunas iniciativas que involucran a la lúdica y sus dinámicas como un activo importante dentro de iniciativas comerciales.

Dos ejemplos de asociaciones simbióticas se pueden apreciar en el caso del sistema de la multinacional de entrenamiento LEGO, y el caso colombiano de la Liga colombiana contra el cáncer.

Un caso importante resulta el Sistema: Lego Serious Play; concebido como una herramienta lúdica diseñada para potenciar la innovación y el rendimiento empresarial. Así, el sistema Lego, se ha convertido en una técnica que mejora la resolución de problemas en grupo, mediante la utilización de las habilidades visuales, auditivas y cinestésicas. En este sistema de resolución de problemas, los participantes manejan un lenguaje común a partir justamente de la neutralidad de las piezas, que se convierten en la forma de expresar sus ideas y concepciones de determinada posición frente a una temática dada.

En Colombia este sistema de entrenamiento lúdico Lego Serious Play, se viene utilizando en la Institución de enseñanza SENA desde hace algunos años y se constituye en un escenario emergente donde el juego y los procesos lúdicos son considerados detonadores de progreso y de innovación empresarial.

Otro caso nacional emblemático de simbiosis comercial y social donde el juego y la recreación se ofrecen como posibilidad de acercamiento entre el consumidor y las entidades comerciales, son los que mantiene la Liga Colombiana contra el Cáncer y distintas empresas, como la fundación Mundo Mujer y la empresa Tena quienes auspician y promueven la carrera de la mujer, una actividad recreativa y deportiva que se realiza anualmente en Colombia. Esta actividad de carácter lúdico, por un lado, tiene un objetivo institucional: el cuidado y la prevención de la salud mediante la realización de acciones lúdicas y deportivas. Y del otro lado; la acción de difusión comercial de las empresas auspiciantes con el fin de fidelizar sus clientes mediante la experiencialidad de sus marcas en una acción lúdica. 
Este tipo de estrategias comerciales cobran cada vez más importancias en los mercados, priorizando el contacto con las personas, sus anhelos y sus emociones. Estas nuevas necesidades son el insumo de las dinámicas de inserción de la lúdica como emergente escenario comercial en el contexto nacional e internacional.

\section{La lúdica y las economías regionales}

El ámbito de aplicación de la lúdica es amplio y heterogéneo. La formulación y ejecución de varias de estas iniciativas provienen de directrices nacionales y se traducen en el contexto regional del sur del país en convocatorias o llamados a revitalizar los circuitos artísticos y culturales propios. Al respecto se debe mencionar que las mecánicas de la lúdica, en todo caso, tienen una incidencia ratificada en los emprendimientos regionales, específicamente en actividades productivas que tienen su génesis en la generación de ideas que luego se transforman en productos, bienes y servicios en el ámbito cultural; esto según la definición del Banco Interamericano de Desarrollo. Esta denominación también atiende las Industrias creativas y todas aquellas manifestaciones e iniciativas artísticas. Así, este tipo de economías creativas comprende los sectores en los que el valor de los bienes y servicios se establece en los derechos de propiedad intelectual, las artes visuales, entornos virtuales de comunicación, artes escénicas, producción editorial, moda, música, publicidad, artesanías, producción audiovisual, diseño colaborativo, investigación y desarrollo de videojuegos y juguetes, software alternativo, TV, radio, y videojuegos, entre otros.

La economía naranja también se ha beneficiado de la evolución de las tecnologías de la información y las comunicaciones; lo que ha permitido su expansión y crecimiento en los últimos años debido a la conectividad. Para el año 2017 este sector representó en Colombia el 13,90\% del Valor Agregado Bruto, según datos del Ministerio de Cultura, presentándose como alternativa de crecimiento y desarrollo de la economía. Los segmentos más representativos dentro de las industrias creativas son los audiovisuales, la educación cultural, la edición de libros y publicaciones periódicas y los juegos y juguetes (ver Figura 2). 


\section{ECONOMÍA NARANJA}

\section{Valor Agregado Bruto Cifras en billones}

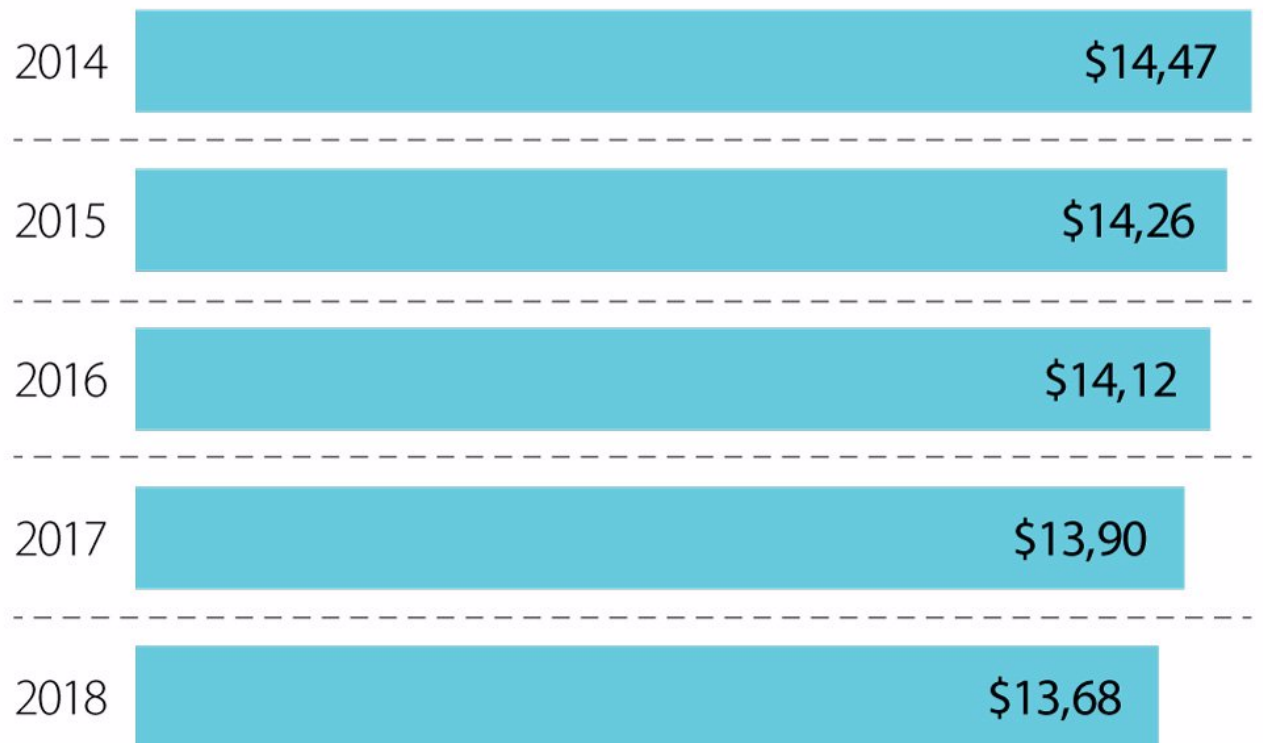

Figura 2. Relación del aporte de la Economía naranja al PIB Nacional de Colombia.

Fuente: DANE. (Periodo 2014-2018).

En Colombia el sector de las Economías naranja en 2012 representó cerca de 1,6 \% del Producto Interno Bruto PIB gracias a dinámicas de crecimiento sostenidas en los últimos años y que para el año 2018 según datos del DANE, se han convertido en más representativas que las ofrecidas por sectores como el de la generación eléctrica y las del cultivo del café que solo aportó el 0,8\% del PIB. Para el año 2018 el ingreso al PIB por concepto de la economía naranja ascendió a los 13,68 billones de pesos. En consecuencia y en vista del crecimiento de este tipo de economías, y el aporte de la lúdica en los procesos mercantiles en el ámbito cultural en Colombia, se promulgó en 2017 la Ley 1834 que establece como una prioridad el fomento a la economía creativa y lleva por nombre Ley naranja. Esta ley promueve una política de incentivos y facilidades de financiación para productores creativos y culturales, y une en ese propósito a los ministerios de Hacienda, del Trabajo, de Educación, de Comercio, del Interior, de Tecnologías de la Información y Comunicaciones, a la Dirección Nacional de Planeación, el DANE, el SENA, la Dirección de Derechos de Autor y Findeter. Además, esta norma 
presenta una estrategia de fortalecimiento a través de las $7 \boldsymbol{i}$ (información, instituciones, industria, infraestructura, integración, inclusión, inspiración) que permiten la integración de instituciones, actores, sectores y mercados. En la actualidad se espera la expedición del Decreto presidencial por el cual se crea y reglamenta el funcionamiento del Consejo Nacional de la Economía Naranja, el cual será el organismo asesor y consultivo del Gobierno Nacional, encargado de formular lineamientos generales de política y de coordinar las acciones interinstitucionales necesarias para la promoción, defensa, divulgación y desarrollo de la economía creativa.

\section{El contexto regional}

En nuestra región se han venido presentando alentadores avances en materia de la incorporación de la lúdica como detonador de distintas iniciativas culturales y comerciales. En este sentido hay que reconocer que en el Plan de Desarrollo 2016-2019 de la Gobernación de Nariño ha considerado el programa de Deporte, Recreación y Actividad Física como una de las prioridades. En este plan de acciones se establece como objetivo la generación de estrategias multisectoriales con calidad técnica, humana y financiera para el desarrollo del deporte social y comunitario; prácticas recreativas para el disfrute del tiempo libre y esparcimiento intelectual, y actividades físicas, como un nuevo marco de relaciones sociales que fomenten la cultura del juego limpio, el pleno desarrollo de las potencialidades del ser humano para su realización y mejoramiento de la calidad de vida individual y social, y la convivencia ciudadana inspirada en el respeto, la libertad y la diversidad.

De la misma forma, la Alcaldía de Pasto en ejecución de su Plan de Desarrollo Municipal Pasto Educado Constructor de Paz 2016-2019, ha considerado las políticas de fomento de la Casa de la Ciencia y el Juego de la ciudad de Pasto. La Casa de la Ciencia y el Juego es un proyecto pedagógico y de comunicación que viene funcionando hace más de10 años, cuyo propósito fundamental es promover, divulgar y recrear el aprendizaje de la ciencia y la tecnología como saberes básicos para desempeñarse con éxito en el presente siglo. La Casa presenta 80 montajes interactivos organizados en diferentes mundos o temas en 400 metros cuadrados y es atendido por personal de la Casa y estudiantes del Liceo de la Universidad de Nariño, quienes prestan su servicio obligatorio. Así mismo, el Plan de la Alcaldía estipula la construcción e implementación de la política pública del deporte, con un capítulo especial de juegos tradicionales y autóctonos del pueblo Quillasinga dado el legado en materia de recreación y deporte de este pueblo ancestral. 
Sumado a estos emprendimientos sociales se pueden sumar los insumos que derivaban del centro CISNA, concebido como una iniciativa comunicacional entre gobierno y ciudadanías a partir de procesos metodológicos que vinculan las mecánicas lúdicas como insumo en la generación de estrategias de difusión y promoción de contenidos.

A este contexto se puede añadir el desarrollo por parte de varias organizaciones privadas y con apoyo de los entes gubernamentales de la Convención Internacional del Comic de Pasto, que se celebra cada año en las instalaciones del Colegio Champagnat. Este evento se ha consolidado con la ampliación continua de los espacios de exhibición, comercialización y de eventos, lo cual demuestra el fuerte impulso que tienen en la actualidad las industrias asociadas a la fantasía, la lúdica y las artes en la región.

Finalmente, otra iniciativa más de consolidación de la lúdica como factor de innovación en las economías emergentes regionales lo representa la reciente aprobación por parte del Ministerio de Educación Nacional del programa de especialización denominado: Diseño de experiencias lúdicas, que se inserta dentro de esta gama de proposiciones de estudio y concepción de la lúdica y sus dinámicas como características de las economías emergentes.

\section{CONCLUSIONES}

El diseño como actividad de prefiguración atiende una gran variedad de ámbitos epistemológicos, áreas de acción, métodos de intervención y mecanismos de solución a problemas en una dinámica creciente que involucra cada vez un mayor número de escenarios de actuación.

En la actualidad el diseño ha superado el estado de la materialidad, tradicionalmente signado al objeto funcional y la imagen comunicativa, para introducirse en el desarrollo de procesos de transformación social. De esta manera han aparecido nuevas perspectivas como el diseño centrado en las personas, diseño social, o el involucramiento del diseño en procesos educativos, empresariales, sociales, culturales, entre muchos otros que ubican al diseño como una disciplina enfocada en la innovación y la cultura. 
El diseño y la lúdica, como dinamizadores sociales, ofrecen características y potenciales que se despliegan también en escenarios comerciales emergentes, donde las mecánicas del juego se involucran evidenciándose como un fenómeno creciente y lleno de posibilidades transdisciplinarias. Así mismo, el estudio del juego desprendido de todas las funciones utilitarias que cada disciplina le adjudica, se convierte en una oportunidad ideal para generar transformaciones en las personas y la sociedad.

En la lúdica y el diseño, insertada en escenarios mercantiles, reposan posibilidades de innovación y cambio social. De esta manera, con la aparición de estos nuevos modelos de la economía naranja emergen nuevos escenarios para que las acciones comerciales y sociales que contemplen la lúdica como acción que potencializa las relaciones sociales e identitarias del individuo, se conviertan en un recurso que se debe trabajar desde procesos de investigación que contemplen el juego como una actividad connatural del ser humano y en esa medida se priorice su devenir en las lógicas mercantiles que encuentran en este territorio oportunidades de innovación y sobre todo la posibilidad de mejora social, a partir del desarrollo de nuevos productos, nuevas formas de comunicación con sus usuarios y nuevas habilidades de competencia y diferenciación comercial, donde la experiencialidad, la emoción y los contextos de uso, serán entre otros, constantes y condicionantes de las nuevas formas de consumo.

\section{REFERENCIAS}

(1) Baudrillard, J., Boudon P, y Moles A. (1974). Los objetos. Buenos Aires. Editorial: Tiempo contemporáneo.

(2) Bbva Game, Disponible en: https://www.bbva.com/es/la-gamificacion-jugar/

(3) Carrera de la Mujer (2018). disponible en: https://www.carreradelamujercolombia.com/tiempoparati.php

(4) Cisna. Centro de innovación social de Nariño. Disponible en http://innovacionsocial.xn--nario-rta.gov.co/

(5) Deterding, S., Dixon, D., Khaled, R., y Nace, L. (2011). Gamification: Toward a definition. Disponible en: http://gamification-research.org/wp-content/uploads/2011/04/02-Deterding-Khaled-Nacke-Dixon.pdf

(6) Dussel, E. (1984). Filosofía de la producción. Bogotá. Ed Clacso.

(7) Huizinga, J. (2012). Homo Ludens. Traducción de Eugenio Imaz. D.F. México. Fondo Mixto de Cultura. Editorial. 
(8) Especialización en Diseño de experiencias lúdicas, (2020). Resolución 008250 del 27 de mayo. Ministerio de Educación Nacional de Colombia. Disponible en https://periodico.udenar.edu.co/men-otorga-registrocalificado-programa-especializacion-experiencias-ludicas-facartes/

(9) González, C., Torres, R. (2012). Diseño \& Consumo en la sociedad Contemporánea. México DF. México. Ed: Designio

(10) Krippenddorf, K. (2016). Rediseñar el diseño una invitación a un futuro responsable. Disponible en: http://infolio.es/articulos/krippendorff/redesign.pdf

(11)La economía creativa. Ley naranja. Ley 1834 de 23 de mayo de 2017, de economía naranja. Disponible en: https://dapre.presidencia.gov.co/normativa/normativa/LEY\%201834\%20DEL\%2023\%20DE\%20MAYO\%20D E\%202017.pdf

(12)Liga Colombiana contra el cáncer. Disponible en: https://www.ligacontraelcancer.com.co/

(13) Messi park (2020). South China Morning Post. Disponible en: https://www.scmp.com/news/china/society/article/2114991/first-look-chinas-lionel-messi-soccer-theme-park

(14) Metodología Lego Serious Play. Disponible en: https://seriousplaylatam.com/index.php/eventosempresariales/lego-serious-play

(15) Ministerio de Cultura Colombia. Porqué es importante la economía naranja en Colombia. Disponible en: https://www.mincultura.gov.co/prensa/noticias/Documents/atencion-alciudadano/_ABC_ECONOMI\%CC\%81A_NARANJA_.pdf

(16) Nike+, (2016). disponible en: https://www.nike.com/xl/es_la/c/nike-plus/running-app-gps

(17) Norman, D. (2005). The Emocional Design. Why we love (or hate) everyday things. New York. Ed. Basic Books.

(18) Piaget, J. (1961). La formación del símbolo en el niño. México: Ed. F.C.E.

(19) Plan de Desarrollo Gobernación de Nariño. Colombia. Disponible en: https://nariño.gov.co/inicio/index.php/gobernacion/plan-de-desarrollo/354-plan-de-desarrollo-departamentalnarino-corazon-del-mundo-2016-2019

(20) Programa: Pasto educado constructor de paz. Disponible en: https://xn--nariorta.gov.co/inicio/index.php/gobernacion/ppasto\%20educado\%20contructor\%20de\%20pazlan-dedesarrollo/354-plan-de-desarrollo-departamental-narino-corazon-del-mundo-2016-2019

(21) Ramírez, C. (2011). Propuesta metodológica para el desarrollo de productos. Rev. Pensamiento \& gestión, 30. Universidad del Norte. pp 21-45.

(22) Rodríguez, J. (2007). 50 Años del Juguete Industrial Colombiano. Trabajo Monográfico de Investigación. Universidad Nacional de Colombia.

(23) Serrano, M. (2013). Design Thinking. Lidera el futuro. Ed. Alfaomega. Cdmx, México.

(24) Simon, H. (2006). Las Ciencias de lo Artificial. Madrid. España. Ed. Comares

(25) The fun theory. Disponible en: https://gestion.pe/blog/consumerpsyco/2014/01/the-fun-theory-la-diversionco.html/ 
(26) Toffler, A. (1980). The Third Wave. La tercera ola. Bogotá Colombia. Ed. Plaza \& Janes. 